\title{
A Comparison of 3D Facial Features in a Population from Zimbabwe and United States
}

\author{
Elvin Bhaskar ${ }^{1}$ Chung H. Kau ${ }^{1}$ \\ ${ }^{1}$ Department of Orthodontics, University of Alabama, Birmingham, \\ Alabama, United States
}

Eur J Dent 2020;14:100-106

\begin{abstract}
Address for correspondence Chung $\mathrm{H}$. Kau, BDS, MSc, MBA, PhD, ABO, Department of Orthodontics, University of Alabama, Suite 305 1919, 7th Avenue South, Birmingham, AL35294, United States (e-mail: ckau@uab.edu).
\end{abstract}

\begin{abstract}
Keywords

- surface acquisition systems

- 3D faces

- 3D imaging

$-3 \mathrm{D}$ analysis

Objectives The purpose of this study was to determine the differences in three-dimensional (3D) facial features in a population from Zimbabwe and the United States. In addition, this study seeks to establish an average facial template of each population allowing clinicians to treat patients according to their cultural esthetic perceptions.

Materials and Methods Three hundred one subjects from Zimbabwe and the United States were carefully selected and recruited for the study. Each subject presented with a normal facial profile, no asymmetries, and normal body mass index. The 3D images were captured using the $3 \mathrm{dMD}$ cameras. All images were further separated into male and female groups of the respective populations and imported to a dedicated software for analysis.

Statistical Analysis The 3D facial images were constructed using Rapidform 6 software to recreate a composite facial average for each group representing a male and female average 3D face of Zimbabwean (Zim) and United States origin.

Results The linear measurements showed that the maximum average distance between the Zim-M and Zim- $F$ was $1.24 \mathrm{~mm}$ and the minimum distance between the African American (AA)-M and AA- $F$ was $0.24 \mathrm{~mm}$. This was the absolute distance. When the signed linear measurements were taken into consideration, the maximum average distance between Zim- $\mathrm{F}$ and AA-M was $1.22 \mathrm{~mm}$ and the least average distance between the Zim-M and AA-M was $0.22 \mathrm{~mm}$. The absolute color histograms showed greatest similarity between the Zim-M and AA-M at 58\% and the Zim-F had a 25 and $27 \%$ similarity with the AA-F and Zim-M, respectively.

Conclusion The Zim-F showed the most variable features with a broader face, prominent forehead, and retruded alar base compared with their male counterparts and the Zim-M showed a wider prominent malar/zygomatic region, and prominent lateral supraorbital regions. There was a high similarity of $58 \%$ between the Zim-M and the AA-M, with the Zim-M showing a more protrusive superciliary arches, and a lateral zygomatic region tapering to the root of the nose.
\end{abstract}

DOI https://doi.org/ 10.1055/s-0040-1702258 ISSN 1305-7456.
(C2020 Dental Investigation Society
License terms

(ㄷ) (1) $\Theta \circledast$ 


\section{Introduction}

Over the years, many methods have been devised to capture three-dimensional (3D) facial morphologies. ${ }^{1-4}$ Most of the time, development ran concurrently with technology and from anthropological studies to X-rays and finally 3D imaging was how development occurred. ${ }^{5-7}$ At present, 2D X-ray imaging is still the mainstay of today's diagnosis, but 3D techniques are rapidly changing these. ${ }^{8,9}$ The study of facial form normally uses lines and angles from a cephalogram. Very few studies have discussed the 3D facial forms of subjects of African descent. ${ }^{10,11}$

\section{African Cephalometric Norms}

Connor and Moshiri in the mid-80s studied cephalograms of Caucasian and African American (AA) people for orthognathic profile preference and found significant variation in various landmarks such as the maxillary and mandibular jaw landmarks, mandibular length, and the Wits appraisal. They performed other soft tissue analysis like teeth display, upper lip length, lower lip length, throat length, and lip chin throat angle. ${ }^{12}$ Another study by Flynn et al showed that AA had more maxillary skeletal prognathism, increased lower facial height, increased skeletal facial convexity, lower incisor proclination, increased upper and lower lip lengths, and increased soft tissue thickness of lips and chin. These subjects also displayed less nasal depth, projection, and smaller nasolabial angle in AA people. ${ }^{13}$ Another study done by O'Reilly investigated soft tissue profile change in AAs after dentoalveolar setback and concluded significant variation in horizontal and vertical lip thickness. ${ }^{14} \mathrm{As}$ late as 2007 Beukes et al studied South African silhouette pictures and evaluated most pleasing and unpleasant profiles and established typical soft tissue values. The most salient feature they noted was upper lip prominence of 5 to $6 \mathrm{~mm}$ more than their AA counterparts; however, other factors such as angular measurements of nose, lip, and chin were in close proximity to those given by Naidoo and Miles. ${ }^{15}$ Last but not least and possibly the most relevant to this study is the work of Dandajena et al who have cephalometrically analyzed dentoalveolar relations and anterior facial heights of the Shona people. He found that anterior facial height was higher in men than in women. The older age groups had shorter anterior face height (AFH) than the younger group. The AFH of the Shona was lower than that of the AAs. All AFHs for the men and only TAFHs for the women were significantly shorter for the Shona than the Caucasians. ${ }^{16}$

Another study by the same author involving the study of lateral cephalograms of 12 angular and 6 linear measurements showed that they had a low Frankfort-mandibular plane angle with a receding chin. Both the maxilla and mandible sella-nasion to A point (SNA) and sella-nasion to B point (SNB) were prognathic and the ANB difference was large. The maxillary incisors were more upright as compared with Caucasian people while measuring the maxillary incisor to NA; however, the lower incisor to mandibular plane angle (IMPA) were relatively proclined at $105.8 \pm 6.0$ degrees and this proclination was considered to be compensatory to the prognathic maxilla. ${ }^{17}$

\section{Soft Tissue Hard Tissue Paradigm}

The specialty of orthodontics has been based on the angle paradigm that involved treating the hard tissues only, getting the teeth into perfect occlusion and allowing soft tissue to follow. The soft and hard tissue are an intricate paired phenomenon, where by one influences the other in an ever constant dynamic interaction. ${ }^{18}$ Teeth usually occupy a neutral zone between muscular intra oral tissues such as the tongue and extra oral soft tissue like the muscles of facial expression and their whole related complex. ${ }^{19}$ Treating only the hard tissues over the years created dental relapse due to displacement from the neutral desired positions. However, the lips in general are also affected by a change in lip thickness and also possible lip lengthening due to the constant pull of gravity. As a result, it is important to consider lip support during extraction versus nonextraction decision. ${ }^{20}$

The specific aims of the study were to determine the average morphology of the Zimbabwean (Zim) people both male and female in a specific age group and compare facial morphological differences of the Zim people to that of AA norms in Birmingham, Alabama, of a similar age.

\section{Materials and Methods}

Subjects were selected from the capital city Harare, Zimbabwe, and Birmingham, Alabama, United States. Both sample sizes were randomly selected from a specific age group from the surrounding metropolitan area.

A questionnaire was handed out to determine demographical origin and other inclusion criteria that include are as follows:

1. Subjects between the age of 18 to 30 years,

2. Ethnicity of Shona descent,

3. Subjects had no adverse skeletal deviations,

4. Subjects with no history of craniofacial deformation,

5. Body mass index within an acceptable reading,

6. No previous orthognathic surgery,

7. No history of orthodontics.

\section{Imaging System}

The 3dMDfaceTM system used for this comprised two infrared cameras and one color camera that casts a random light pattern on the object. The image was captured from a set series of angles to represent the image in the format of a 3D shell. Capture time was 1.5 milliseconds with an accuracy of $1.5 \%{ }^{21}$

\section{Image Acquisition}

All subjects rested their head position in a natural way because it is the most attainable and reproducible. The subjects sat on a chair that could be adjusted for the right position and were asked to gaze into a mirror set in front of them. Their eyes were levelled, by looking into the mirror and stool adjusted according to their heights as required. The subjects were told to swallow and keep jaws relaxed just before image capture.

\section{Processing of Facial Shells}

All images imported to Rapidform software 2006, INUS Technology, Seoul, Korea (RF6) for analysis. Areas such as the hair, ears, neck, and shoulders were removed by a computer tool and the surface defects filled in. One facial shell for each individual was created. ${ }^{22}$ 


\section{Average Face Construction}

Each subject's shell was carefully created and they were aligned and to the "best-fit algorithm." Subsequently, the shells were averaged by a computer algorithm that produced an average shell, one for the females and the other for males.

The steps required to produce and average are summarized as follows:

1. All images were aligned to form the principal axis of rotation, also known as the center of mass.

2. Manual alignment can also be used to improve the position.

3. The built-in "algorithm" in RF6 calculated the best fit for the shells precise registration.

4. The 3D datapoints of the images were averaged based on a facial template.

5. The resulting output of the average algorithm gave a point cloud reading, which was then mathematically triangulated to obtain an average face.

6. Filling in or snubbing any mesh defects improves the average faces.

7. A color texture was applied onto the facial shell. In addition, shells with one positive and one negative deviation were created.

\section{Parameters Measured}

Two average facial shells were generated: Zimbabwean male (Zim-M), Zimbabwean female (Zim-F). These shells were carefully aligned on each other using the RF6 software. A specialized technique to compare morphological differences was used. This process comprised manually aligning five points of the facial scans, two points on outer canthus of both eyes and two points on the inner canthus of both eyes, and one point on the tip of the nose. The RF6 software then determines the best fit of the two shells.

The parameters used to study these results were as follows:

1. Linear measurements in millimeter, both absolute and signed, which measure the greatest difference between and within the shells, respectively.

2. Color histograms as a percentage, which show similarities, positives and negatives.

3. Surface areas and shapes, which show range of distribution.

\section{Linear Measurements}

The differences are measured by a linear measurement at any given point of the facial topography. The linear difference is the discrepancy between the two shells and the value is used to quantify the deviation. A percentage similarity can also be calculated.

\section{Color Histograms}

The two shells are compared with each other with one being the base and the other a superimposition. A positive difference is noted if the superimposed shell is more positive than the base and a negative difference is noted if the superimposed shell is more deficient than the base. The differences are identified by different color depictions: black indicating similarity between the shells, red indicating a positive change (prominence), and blue indicating a negative change (deficient). This allows us to distinguish and analyze surface topography and its differences.

\section{Surface Area and Shapes}

This is automatically generated by the RF6 software with a tolerance level of $0.50 \mathrm{~mm}$ that was offset to the paired surface shells. The value here was obtained from previous work that shows that $90 \%$ of created composite scans are within the $0.85 \mathrm{~mm}$ error. Any variations within $0.50 \mathrm{~mm}$ were considered to be similar surfaces, while surface areas outside this tolerance showed up as a color map of blues and reds.

\section{Results}

The final sample was 301 subjects. These included 201 people: 107 men and 94 women from Zimbabwe. Average faces were constructed for each of the groups and compared with each other and to that of AAs in Birmingham, Alabama (50 men and 50 women) (-Figs. $\mathbf{1}$ and $\mathbf{2}$ ).

\section{Linear Measurements}

The absolute linear measurement in gender differences ranged from 0.42 (AA-M vs. AA-F) to $1.24 \mathrm{~mm}$ (Zim-F vs. Zim-M) as shown in - Table 1. Further differences between
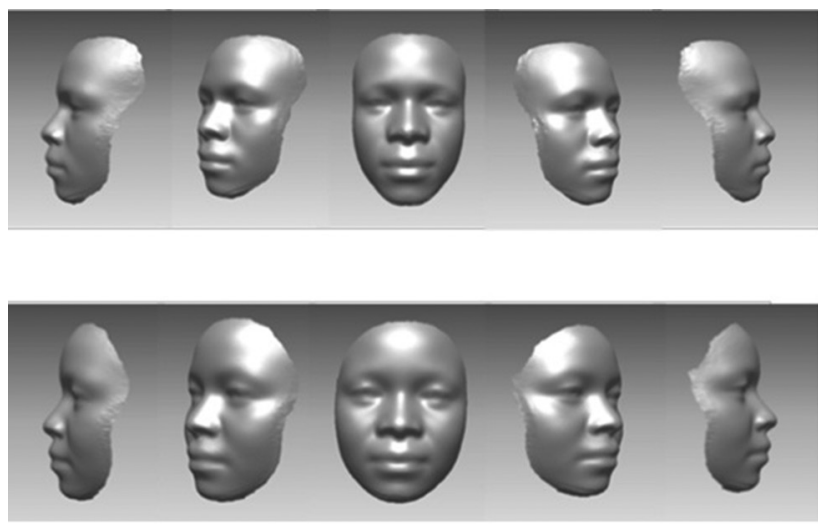

Fig. 1 Average facial constructions for the Zimbabwean males (row 1) and the Zimbabwean females (row 2).
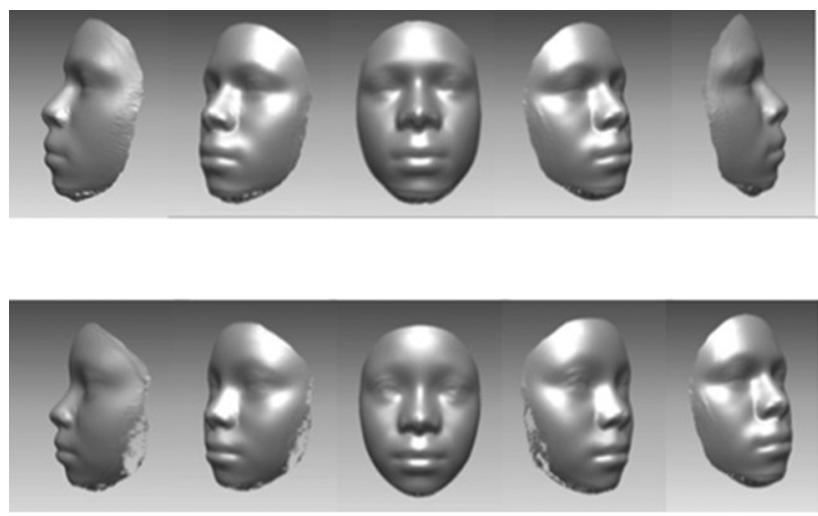

Fig. 2 Average African American males (row 1) and African American females (row 2). 
Table 1 Absolute linear measurements indicating differences between facial shells

\begin{tabular}{|l|l|l|l|}
\hline & Average distance $(\mathbf{m m})$ & Standard deviation (mm) & Maximum distance (mm) \\
\hline Zim-M vs. Zim-F & 1.24 & 1.20 & 5.09 \\
\hline AA-F vs. Zim-F & 1.16 & 0.87 & 4.21 \\
\hline Zim-F vs. AA-M & 1.22 & 0.96 & 4.47 \\
\hline Zim-M vs. AA-M & 0.51 & 0.41 & 2.02 \\
\hline AA-F vs. Zim-M & 1.04 & 0.92 & 5.01 \\
\hline AA-F vs. AA-M & 0.42 & 0.35 & 2.99 \\
\hline
\end{tabular}

Abbreviation: AA, African American.

Table 2 Signed color map measurement indicating differences in facial shells

\begin{tabular}{|l|l|l|l|}
\hline & Average distance $(\mathrm{mm})$ & Standard deviation (mm) & $\begin{array}{l}\text { Percentage } \\
\text { Similarity }\end{array}$ \\
\hline Zim-M vs. Zim-F & 0.35 & 1.62 & 27.08 \\
\hline AA-F vs. Zim-F & 0.39 & 1.40 & 25.79 \\
\hline Zim-F vs. AA-M & 0.55 & 1.45 & 28.79 \\
\hline Zim-M vs. AA-M & 0.02 & 0.66 & 58.75 \\
\hline AA-F vs. Zim-M & 0.04 & 1.39 & 36.19 \\
\hline AA-F vs. AA-M & -0.07 & 0.54 & 57.57 \\
\hline
\end{tabular}

Abbreviation: AA, African American.

Zim and AA are listed below. The maximum distance of $5.09 \mathrm{~mm}$ was seen in (Zim-F vs. Zim-M), while the minimum of $2.02 \mathrm{~mm}$ was seen in (Zim-M vs. AA-M).

\section{Color Histograms}

The differences in color histograms between Zim-M and Zim-F are shown in - Table 2. The \% similarity is $27.08 \%$. The greatest amount of similarity is seen between the Zim-M versus AA-M at 58.75\%, which is quite significant. The AA-M versus AA-F too had a high similarity of $57.57 \%$. The Zim-F sample seems to be the most dissimilar group when compared with their male counterparts and also to that of the AA-M and AA-F. The results in the signed color histograms show the similarities between the same sex individual of different ethnicities but very dissimilar among their own ethnic groups (-Figs. 3-8).
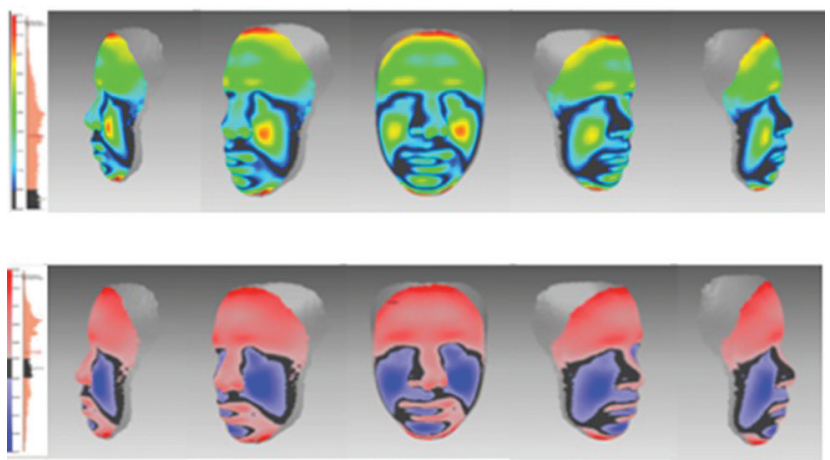

Fig. 3 Absolute and signed histograms of Zim-M versus Zim-F. The differences indicate that average shells are uniquely different and represent each individual group. Zim-F, Zimbabwean females; Zim-M, Zimbabwean males.

\section{Discussion}

Very few studies on 2D analysis have been done on native southern African people, let alone 3D. Isiekwe et al studied the nose prominence relative to other structures of an adult Nigerian population, using the Holdaway analysis. ${ }^{23} \mathrm{~A}$ few photogrammetric studies of North African countries, such as Senegalese students versus Moroccans students where linear measurements were taken to quantify their differences. ${ }^{24}$ Another Sudanese study used a hand laser scanner in 653 subjects and analyzed 14 landmarks on the facial soft tissue. ${ }^{25}$ Soft tissue cephalometric studies on Nigerians, Ghanaians, and Sudanese teenagers were done to determine soft tissue pattern
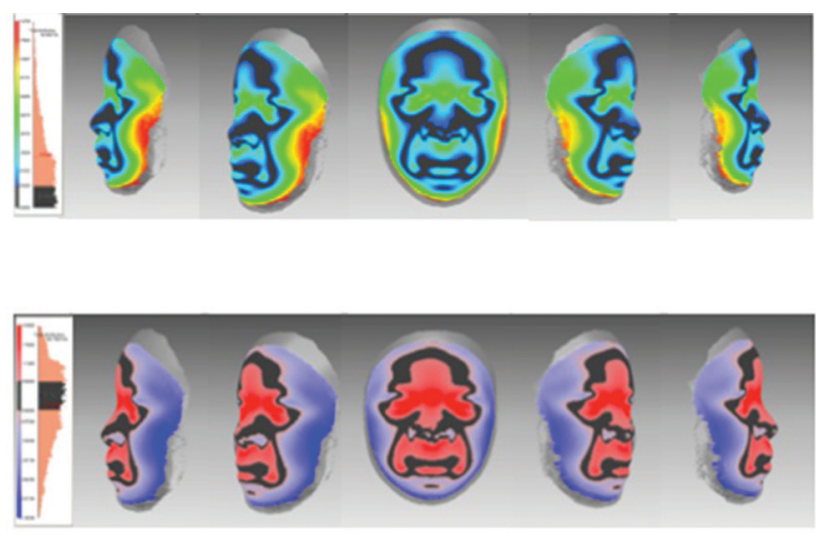

Fig. 4 Absolute and signed histogram of AA-F versus Zim-F. The average distance of the differences was $1.16 \pm 0.87 \mathrm{~mm}$. The absolute difference ranged from 0 to $4.21 \mathrm{~mm}$. Positive differences are seen in red and negative differences in blue. The average difference was $0.39 \pm 1.40 \mathrm{~mm}$ and $25.79 \%$ of the faces were similar. AA-F, African American females; Zim-F, Zimbabwean females. 

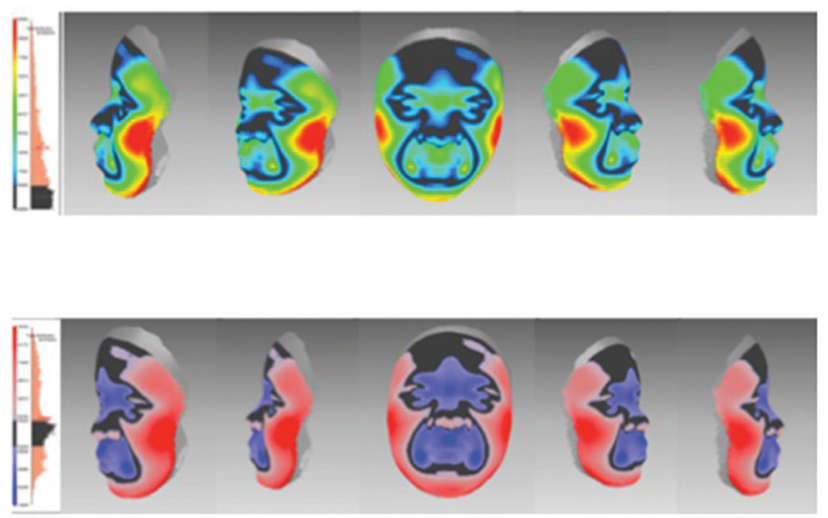

Fig. 5 Absolute and signed histogram of Zim-F versus AA-M. AA-M, African American males; Zim-F, Zimbabwean females.
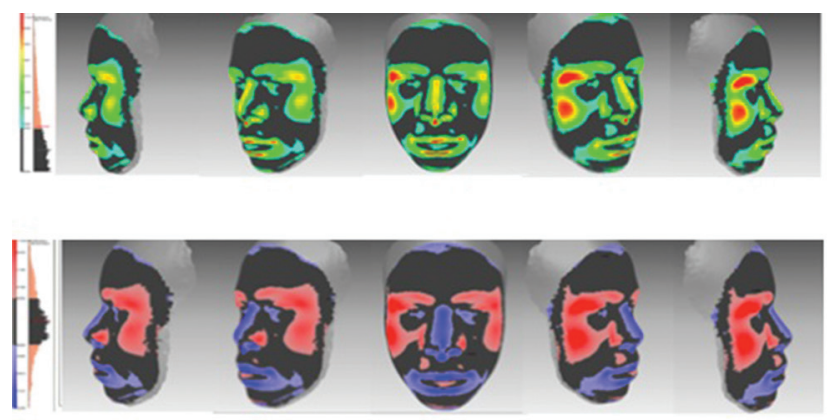

Fig. 6 Absolute and color histogram of Zim-M versus AA-M. The average distance of the differences was $0.51 \pm 0 \pm .41 \mathrm{~mm}$. The absolute difference ranged from 0 to $4.47 \mathrm{~mm}$. Positive differences are seen in red and negative differences in blue. The average difference was $0.02 \pm 0.66 \mathrm{~mm}$ and $58.75 \%$ of the faces were similar. AA-M, African American males; Zim-M, Zimbabwean males.
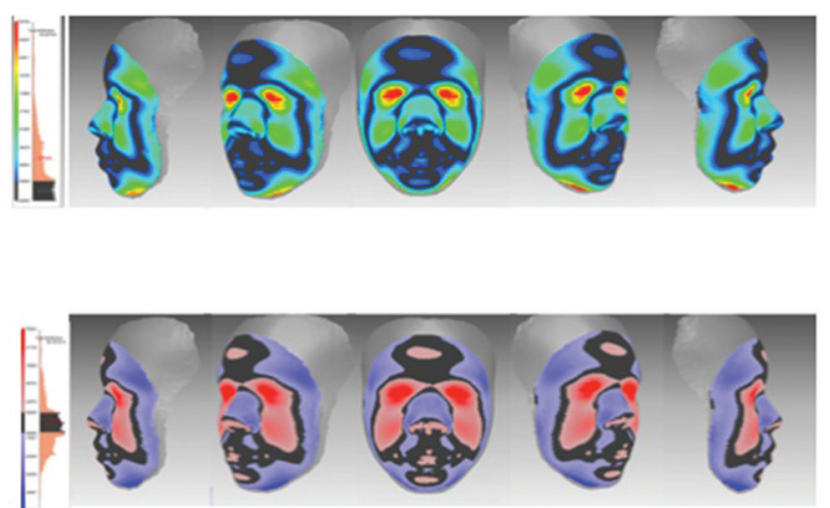

Fig. 7 Absolute and color histogram of AA-F versus Zim-M. AA-F, African American females; Zim-M, Zimbabwean males.

and compared with that of Caucasian counterparts. Significant statistical difference was apparent. ${ }^{26}$ Only one soft tissue profile study was done in South Africa to establish a profile index for bimaxillary protrusion and soft tissue preference..$^{15}$

\section{Zimbabwean Male and Female Morphology}

When looking at the color histograms, the Zim-M show a more protrusive forehead, supraorbital, and nasal bridge
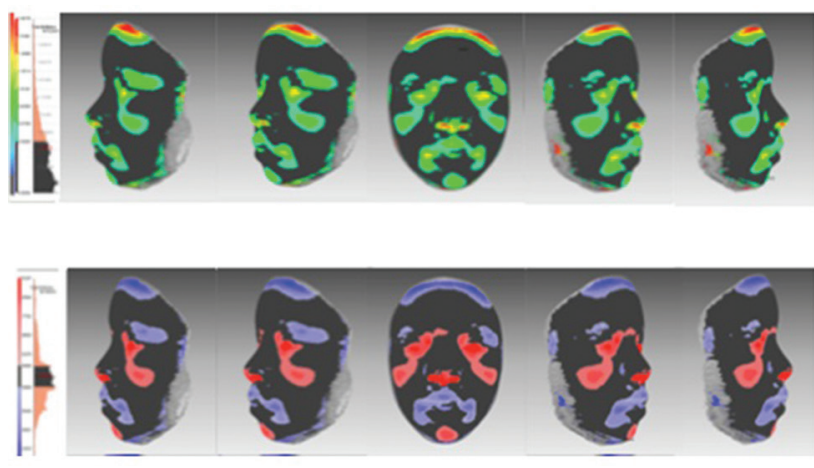

Fig. 8 Absolute and color histogram of AA-F versus AA-M. The differences indicate that average shells are uniquely different and represent each individual group. AA-F, African American females; AA-M, African American males.

area while being retrusive in the infraorbital and lateral alar region. They also showed accentuated perioral region. The similarities between the two sexes are the lateral parts of the nose, subnasal area superior portion of the lip extending down to the corner of the mouth toward the parasymphysis area.

\section{Comparison between Population Subgroups}

When comparing histograms of the same sexes but of different population groups, the similarities for the males were considerably higher than the females. The similarities were in the inner canthus of the eyes, lateral nasal region, submental and lower borders of the chin. The noticeable difference between them were nasal and perioral areas that showed a more retrusive region, while the malar/zygomatic and lateral periorbital ridges were more prominent. The females between the two subgroups were more dissimilar with the forehead, inner canthus of the eyes, nasal bridge, upper and lower lips being more prominent, while the lateral aspects of the face that is the malar region extending down to the mandible seem more retrusive.

\section{Clinical Implications}

It is apparent that all along we have been diagnosing and categorizing various ethnic groups into a general pool of Caucasian norms..$^{27-29}$ The current trend of treating facial profile first and dentition to follow has taken great precedence. Understanding soft tissue norms first and then comparing them to other average shells give us a perspective on variations. This allows us to plan incisor positions according to average profiles for that age group and it also gives us a basis to form data on which we can build a foundation to understand trends among ethnicities.

It is obvious in this study that despite the similarity in African descent in the two populations that significant differences do exist for each of the facial averages. It is, therefore, important as always to create normative data for each individual population or race and to make it also specific to gender.

This study uses 3D averages to differentiate between two distinct populations. It uses new technologies for the better understanding of facial differences and represents a new way to quantify populations. While previous studies have been 
used in AAs, ${ }^{10,11}$ this is the first time this type of analysis is used. Furthermore, in recent times, genomic studies ${ }^{29,30}$ have also been introduced to better quantify data obtained from 3D faces and these will play a more significant role in the era of 3D facial imaging. ${ }^{31}$

\section{Conclusion}

The following conclusions may be drawn from this study.

1. When compared with the AA-M, the Zim-M have a prominent zygomatic, lateral supraorbital, and infraorbital region. Bridge of the nose and perioral area around the lips were also retrusive.

2. When compared with the AA-M, the Zim-M show a more protrusive forehead, supraorbital, and nasal bridge area while being retrusive in the infraorbital and lateral alar region. They also showed accentuated perioral region.

3. When compared with AA-M, the Zim-M show wider supraorbital regions but smaller more retruded perioral regions.

4. When compared with AA-F, the Zim-F show a subtler malar region with less prominent lips.

5. When compared with Zim-F, the AA-F are more protrusive in the middle forehead area, alar base, and lateral perioral region, retrusive in the lateral zygomatic region, gonial, and submental area.

\section{Conflict of Interest}

None declared.

\section{References}

1 Gor T, Kau CH, English JD, Lee RP, Borbely P. Three-dimensional comparison of facial morphology in white populations in Budapest, Hungary, and Houston, Texas. Am J Orthod Dentofacial Orthop 2010;137(3):424-432

2 Palomo JM, Kau CH, Palomo LB, Hans MG. Three-dimensional cone beam computerized tomography in dentistry. Dent Today 2006;25(11):130, 132-135

3 Bo Ic M, Kau CH, Richmond S, et al. Facial morphology of Slovenian and Welsh white populations using 3-dimensional imaging. Angle Orthod 2009;79(4):640-645

$4 \mathrm{Kau} \mathrm{CH}$. Creation of the virtual patient for the study of facial morphology. Facial Plast Surg Clin North Am 2011;19(4):615-622, viii

5 Celebi AA, Kau CH, Ozaydin B. Three-dimensional anthropometric evaluation of facial morphology. J Craniofac Surg 2017;28(5):e470-e474

6 Kim JY, Kau CH, Christou T, Ovsenik M, Guk Park Y. Three-dimensional analysis of normal facial morphologies of Asians and whites: a novel method of quantitative analysis. Plast Reconstr Surg Glob Open 2016;4(9):e865

7 Lightheart KG, English JD, Kau CH, et al. Surface analysis of study models generated from OrthoCAD and cone-beam computed tomography imaging. Am J Orthod Dentofacial Orthop 2012;141(6):686-693

8 Kau CH, Richmond S. Three-dimensional analysis of facial morphology surface changes in untreated children from 12 to 14 years of age. Am J Orthod Dentofacial Orthop 2008;134(6):751-760
9 Kau $\mathrm{CH}$, Richmond S, Incrapera A, English J, Xia JJ. Three-dimensional surface acquisition systems for the study of facial morphology and their application to maxillofacial surgery. Int J Med Robot 2007;3(2):97-110

10 Kau CH, Wang J, Davis M. A cross-sectional study to understand 3D facial differences in a population of African Americans and Caucasians. Eur J Dent 2019;13(4):485-496

11 Talbert L, Kau CH, Christou T, Vlachos C, Souccar N. A 3D analysis of Caucasian and African American facial morphologies in a US population. J Orthod 2014;41(1):19-29

12 Connor AM, Moshiri F. Orthognathic surgery norms for American black patients. Am J Orthod 1985;87(2):119-134

13 Flynn TR, Ambrogio RI, Zeichner SJ. Cephalometric norms for orthognathic surgery in black American adults. J Oral Maxillofac Surg 1989;47(1):30-39

14 O'Reilly MT. Integumental profile changes after surgical orthodontic correction of bimaxillary dentoalveolar protrusion in black patients. Am J Orthod Dentofacial Orthop 1989;96(3):242-248

15 Beukes S, Dawjee SM, Hlongwa P. Soft tissue profile analysis in a sample of South African Blacks with bimaxillary protrusion. SADJ 2007;62(5):206-212, 208-210, 212

16 Dandajena TC, Chung KW, Nanda RS. Assessment of anterior face height in a native African sample. Am J Orthod Dentofacial Orthop 2006;130(2):196-201

17 Dandajena TC, Nanda RS. Bialveolar protrusion in a Zimbabwean sample. Am J Orthod Dentofacial Orthop 2003;123(2):133-137

18 Arnett GW, Jelic JS, Kim J, et al. Soft tissue cephalometric analysis: diagnosis and treatment planning of dentofacial deformity. Am J Orthod Dentofacial Orthop 1999;116(3):239-253

19 Proffit WR. Muscle pressures and tooth position: North American whites and Australian aborigines. Angle Orthod 1975;45(1):1-11

20 Caplan MJ, Shivapuja PK. The effect of premolar extractions on the soft-tissue profile in adult African American females. Angle Orthod 1997;67(2):129-136

21 Kau CH, Zhurov A, Scheer R, Bouwman S, Richmond S. The feasibility of measuring three-dimensional facial morphology in children. Orthod Craniofac Res 2004;7(4):198-204

22 Kau CH, Richmond S, Savio C, Mallorie C. Measuring adult facial morphology in three dimensions. Angle Orthod 2006;76(5):773-778

23 Isiekwe GI, daCosta OO, Utomi IL, Sanu OO. Holdaway's analysis of the nose prominence of an adult Nigerian population. Niger J Clin Pract 2015;18(4):548-552

24 Diouf JS, Ngom PI, Fadiga MS, et al. [Sagittal photogrammetric evaluation of the soft tissue profile between two different racial groups: a comparative study]. Odontostomatol Trop 2015;38(150):5-14

25 Sforza C, Dolci C, Tommasi DG, Pisoni L, De Menezes M, Elamin F. Three-dimensional facial distances of Northern Sudanese persons from childhood to young adulthood.J Craniomaxillofac Surg 2014;42(5):e318-e326

26 Fadeju AD, Otuyemi OD, Ngom PI, Newman-Nartey M. A study of cephalometric soft tissue profile among adolescents from the three West African countries of Nigeria, Ghana and Senegal. J Orthod 2013;40(1):53-61

27 Wirthlin J, Kau CH, English JD, Pan F, Zhou H. Comparison of facial morphologies between adult Chinese and Houstonian Caucasian populations using three-dimensional imaging. Int J Oral Maxillofac Surg. 2013;42(9):1100-1107

28 Liu Y, Kau CH, Talbert L, Pan F. Three-dimensional analysis of facial morphology. J Craniofac Surg. 2014;25(5):1890-1894

29 Weinberg SM, Raffensperger ZD, Kesterke MJ, et al. The 3D facial norms database: part 1. A web-based 
craniofacial anthropometric and image repository for the clinical and research community. Cleft Palate Craniofac J 2016;53(6):e185-e197

30 Shaffer JR, Orlova E, Lee MK, et al. Genome-wide association study reveals multiple loci influencing normal human facial morphology. PLoS Genet 2016;12(8):e1006149
31 Zacharopoulos GV, Manios A, Kau CH, Velagrakis G, Tzanakakis $\mathrm{GN}$, de Bree E. Anthropometric analysis of the face. J Craniofac Surg 2016;27(1):e71-e75 